\title{
LA INCIDENCIA DEL MERCHANDISING EN EL CONTEXTO MUNDIAL
}

\section{RECURRENCE OF MERCHANDISING IN THE GLOBAL CONTEXT}

\author{
Mónica Eugenia Peñalosa Otero*, Emigdio Larios Gómez ${ }^{\star *}$, \\ Julio Enrique Lora Suárez ${ }^{\star \star *}$ Jorge Antonio David Díaz ${ }^{\star \star \star \star}$
}

*Maestra en Mercadeo Agroindustrial. Profesora Asociada II. Universidad Jorge Tadeo Lozano, Colombia. ORCID: http://orcid.org/0000-0002-2208-9224

${ }^{\star *}$ Doctor en Ciencias Administrativas. Profesor-Investigador, Visitante Titular en Universidad Jorge Tadeo Lozano, Colombia. Email: herr.larios@gmail.com

ORCID: http://orcid.org/0000-0002-3514-1319

***Maestro en Administración. Profesor Asociado II. Universidad Jorge Tadeo Lozano, Colombia. Email: julioe.lora@utadeo.edu.co. ORCID: http://orcid.org/0000-0003-0889-8217

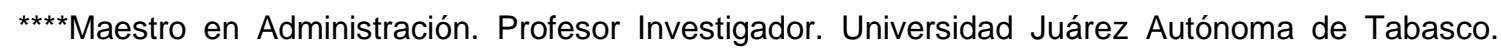
Email: jdavidd@hotmail.com. ORCID: http://orcid.org/0000-0002-8464-7703

Dirección para recibir correspondencia: monica.penalosa@utadeo.edu.co 


\section{RESUMEN}

OBJETIVO: Mostrar la importancia de esta técnica, pasando por los autores que han aportado a la construcción del concepto haciendo ver que lo más importante es convertir la compra en una verdadera experiencia que genere un momento inolvidable para el cliente y que esto redunde en ventas y rentabilidad para la compañía; todo lo anterior, toma el nombre de Merchandising.

MATERIAL Y MÉTODO: El tipo de estudio se clasifica como exploratorio, con el uso de la técnica análisis de contenido, según el cual examinamos un tema poco estudiado o del cual se tiene un bajo conocimiento.

RESULTADOS: Con esta revisión de la literatura, se aprecia la importancia de consolidar los elementos que desde la teoría pueden tener relevancia práctica y ser desarrolladas por las empresas en los diversos entornos donde esta opere, permitiendo tener un impacto positivo.

CONCLUSIONES: En muchos aspectos, la importancia del Merchandising radica en su capacidad de manipular psicológicamente al cliente. En última instancia, más allá de la comida, agua y ropa, ningún producto que se encuentre en un establecimiento comercial constituye una necesidad para el cliente. Cuando se obtiene el éxito, el impacto psicológico de las unidades de Merchandising lleva a las ventas, genera conocimiento del producto, crea un entorno visual confortable para el cliente y bombea dinero en el punto de venta. También puede afectar a la forma de ver ciertos productos, influyendo así en las futuras decisiones de compra.

PALABRAS CLAVE: Merchandising. Consumidor. Producto. Surtido. Neuromarketing. 


\section{ABSTRACT}

OBJECTIVE: To show the importance of this technique, from authors who have collaborated with the construction of the concept and who emphasized the importance of making a sale a real experience that generates a memorable experience which in return will translate into sales and profitability for a company or also known as merchandising.

MATERIAL AND METHOD: This is an exploratory study carried out with the content analysis technique, used when it is a new topic or barely studied problem.

RESULTS: With this literature review, it is intended to consolidate the elements of this theory, their pragmatic implications and if implemented in companies, there will be a positive impact in any environment.

CONCLUSIONS: In many aspects, the importance of Merchandising lies in its capacity to psychologically manipulate consumers. Beyond clothes, food, and water, no product is considered a basic need for consumers in a commercial establishment. When success is obtained, the psychological impact of the units leads to more sales, it generates product knowledge, a comfortable visual environment as well as money in a point of sale. It can also can affect the way certain products are perceived, thus influencing future shopping decisions.

KEY WORDS: Merchandising. Consumer. Product. Variety. Neuromarketing.

\section{INTRODUCCIÓN}

El Merchandising, no es otra cosa que el mercadeo llevado al punto de venta. La esencia del Merchandising se centra en dar al producto un papel activo en la venta, por medio de su presentación y entorno, con la finalidad de que pueda venderse por sí solo, sin necesidad de ayuda alguna. El concepto de Merchandising y el de autoservicio forman un todo indisoluble. El Merchandising ha tenido un largo camino desde que 1852, en París, aparecen los Almacenes Bon Marché.

Hace más de un siglo y medio, Arístides Boucicaut, un visionario del comercio, se da cuenta que el comprador de la Capital de Francia ha cambiado, que se ha sofisticado lo suficiente para requerir una nueva forma de comprar, donde lo que se dé sea más atención y productos. El consumidor parisino requería algo más que buenos productos y excelente servicio. 


\section{LA INCIDENCIA DEL MERCHANDISING EN EL CONTEXTO MUNDIAL}

Arístides entiende perfectamente que para ser exitoso se debe dar al consumidor una experiencia agradable, diferente y duradera cuando compra en un local comercial.

Por eso, contra la opinión de muchos, se orienta a un comprador sofisticado, de alto nivel de ingresos, al cual le ofrece algo nunca antes visto en el mundo. Sus tiendas se expanden a toda Europa y son un éxito increíble. En la actualidad, los Almacenes Bon Marché, se han adaptado a los tiempos que corren, y venden por Internet utilizando la tecnología para seguir dando momentos de intensa placidez al consumidor. El concepto, a partir de 1852, ha sido también, exitoso para enorme cantidad de empresas por el mundo entero, que lo han ido adoptado. El autoservicio es hoy día tan corriente que cuando vamos al supermercado no nos damos cuenta que estamos comprando de una forma muy diferente a nuestros abuelos. La nueva forma de comercio, que impulsa el concepto de Merchandising, hace cambios en el consumidor, el productor, el producto, la forma de vender y el vendedor.

MATERIALES Y MÉTODOS: La presente investigación es de tipo exploratoria desde un enfoque documental-bibliográfico, basada en el método cualitativo (Kerlinger, 2002), con enfoque fenomenológico sistémico (Martínez, 2006). La revisión literaria se realizó con base en el análisis de las bases de datos disponibles, orientado a comprender los marcos codificadores e interpretativos de un fenómeno de interés, que en este caso se trata del análisis teórico del Merchandising.

\section{RESULTADOS}

\section{El Merchandising}

El término Merchandising es un vocablo anglosajón que viene del sustantivo merchandis que significa mercancía y con terminación del radical ing., que lleva implícita la idea de acción de movimiento, que por lo general no se presenta una palabra en el habla hispana que represente este término (mercadeando). Existen definiciones de la palabra Merchandising, se nombran algunas de las más importantes. Para la Academia Francesa de Ciencias Comerciales, el Merchandising es una parte del marketing que engloba las técnicas comerciales que permiten presentar al posible comprador el producto o servicio que se quiere vender, en las mejores condiciones materiales y psicológicas. Tiende a sustituir la presentación pasiva del producto o servicio por una presentación activa recurriendo a todo lo que le puede hacer más atractivo: presentación, fraccionamiento, envasado, exposición, decoración. Según Kotler (2006), el Merchandising es el conjunto de actividades realizadas a nivel detallista con el objetivo de: (AIDA) A: Atraer la atención. I: Interesar al cliente D: Despertar el deseo A: Accionar la compra 


\section{LA INCIDENCIA DEL MERCHANDISING EN EL CONTEXTO MUNDIAL}

La American Marketing Association, define el Merchandising como un conjunto de técnicas basadas principalmente en la presentación, rotación y la rentabilidad, comprendiendo un conjunto de acciones llevadas a cabo en el punto de venta destinadas a aumentar la rentabilidad, colocando el producto en el lugar, durante el tiempo, en la forma, al precio y en la cantidad más conveniente. Según Fresco (1999), "son todas las actividades que se llevan a cabo en el punto de venta con el objetivo de lograr que la mercadería expuesta sea elegida y adquirida por el consumidor". Por consiguiente, el Merchandising es el conjunto de estudios y técnicas de aplicación llevados a la práctica, de forma conjunta o separada, por distribuidores y fabricantes, con el objeto de aumentar la rentabilidad en el punto de venta y dar mayor salida a los productos mediante una permanente adaptación del surtido a las necesidades del mercado y la presentación adecuada de las mercancías. El Merchandising surge como consecuencia del desarrollo del autoservicio, de los diferentes tipos de compra, y de los variados comportamientos del consumidor, de la separación del acto de la compra, del acto del pago (ilusión de gratuidad), y del acto del consumo; es decir, se ocupa del acto de la compra. De la misma forma, nace como consecuencia natural de la profesionalización y racionalización del sector minorista, como sector económico de gran importancia en las economías modernas. El marketing es una disciplina enfocada al consumidor, mientras que el Merchandising está totalmente centrado en el punto de venta, en el establecimiento; es el movimiento de la mercancía hacia el consumidor en el punto de venta.

Con el Merchandising, se transmite el posicionamiento de marketing y de los productos al consumidor en el punto de venta. Para comercializar mejor los productos, se usa la promoción para vender en mayor cantidad y el Merchandising para acelerar la venta. El objetivo primordial es el de conseguir y mantener la máxima productividad del espacio y mejorar los resultados a través de una gestión profesional del punto de venta. El foco de interés es el espacio físico del establecimiento. Para Mouton (2003), este objetivo se consigue dando satisfacción al consumidor en el punto de venta, e incrementando la circulación de clientes en el establecimiento.

En estos momentos, el Merchandising concierne a todo tipo de establecimientos, sean en régimen de autoservicio, o no, por lo que es un conjunto de acciones. Sánchez, (1997), afirma que el concepto de Merchandising está muy ligado a los productos de consumo masivo y los autoservicios; es decir, establecimientos donde no hay vendedores y los productos deben venderse por sí mismos. En este sentido, cabría preguntarse si realmente puede hablarse de acciones de Merchandising en el sector bancario que, evidentemente, dista mucho de parecerse a un autoservicio donde los productos se encuentran de cara al público. Sin embargo, los bancos utilizan muchas técnicas de Merchandising en el interior de sus 


\section{LA INCIDENCIA DEL MERCHANDISING EN EL CONTEXTO MUNDIAL}

negocios, tanto para dar a conocer sus productos, como para crear un ambiente agradable de cara al consumidor. Asimismo, Teruel (1995) supone que a través del Merchandising y del marketing directo, se busca otro tipo de relación con el cliente. Símbolos, imágenes, colores, son otros componentes con los que tenemos que contar para que la clientela se identifique, donde la distribución de puestos juega un papel importante a la hora de comercializar diferentes tipos de productos y servicios, en función de qué tipo de segmento se trate.

El origen del Merchandising, se encontró desde las primeras manifestaciones visuales en el contexto de la prehistoria, las cuales son las figuras rupestres de hace más de 25.000 años. El artista del paleolítico utilizaba el símbolo incipiente de la invocación. En Roma, es donde nace la exhibición de mercancías en aparadores o escaparates este procedimiento llevaba a los tenderos a vaciar los establecimientos con tal de exhibir sus mercancías. Se practicaba el Merchandising, desde que el antiguo camellero que, para vender mejor sus naranjas, las colocaba en el suelo en forma de pirámide, y para suscitar el deseo, cortaba por la mitad las más bonitas. También con la pulpa de fruta, cuyas gotas brillaban con el sol, atraían al comprador sediento. Luego con la evolución del comercio al principio, antes incluso de que existieran las tiendas, la mercancía ya se exponía, en el suelo o sobre tablas en los mercados al aire libre. El vendedor atraía al comprador mostrando la mercancía. Después, rápidamente llegaron las tiendas en donde el comprador tenía que pedir el artículo que quería. Entonces el vendedor se iba a la trastienda, buscaba la pieza que se le había pedido, la ponía sobre el mostrador con una o dos más y alababa la solidez de ésta (Wellhoff y Masson, 1999).

En 1852, se produjo la gran revolución del comercio con la llegada de grandes tiendas, inventadas por Aristide Boicicaut. Los productos, situados detrás del vendedor, dan un gran salto por encima de su cabeza para disponerse en estanterías, en contacto directo con los compradores, que circulaban libremente. Se constató que el producto se acerca considerablemente al comprador, disponiéndose al alcance de su mano, lo puede tocar; la acción del vendedor sigue siendo importante, para conocer sobre la mercancía y la libre circulación y las grandes superficies de venta pasan a ser algo habitual. Hacia 1934 en Francia y 1928 en Estados Unidos, se da una segunda evolución: se trata de la "tienda popular" llamada también de precios únicos, derivada de una gran tienda, simplifica sus cuatro principios: Libre circulación en una superficie más modesta, número de referencias limitado, rotación rápida de stocks, con un margen reducido y venta y cobros simplificados.

Actualmente, con la venta moderna, la finalidad lógica de esta evolución es el libre servicio, simplificando un poco más este proceso, el producto se pone directamente en la cesta de compra, por el propio comprador, sin pasar antes por un acto de venta. El comprador pasea 


\section{LA INCIDENCIA DEL MERCHANDISING EN EL CONTEXTO MUNDIAL}

con total libertad, escoge lo que quiere y lo pone en su carro, de esta manera hay una desaparición total del vendedor; libertad total de elección sin ninguna intervención humana. Esta es lógica del Merchandising. De acuerdo con Wellhof y Masson, (1999) "actualmente el producto tiene que venderse sólo. Es un universo de libre elección, el consumidor tiene que aprender a descubrir la oferta y elegir el producto". Para Kepner (1992), el Merchandising debe ser el producto adecuado, en el lugar adecuado, en el momento adecuado, al precio adecuado y en las cantidades adecuadas, con la aplicación de estos cinco lineamientos, que son los requisitos a cumplir para quienes se encargan de trabajar esta técnica. El Merchandising es, ante todo, un buen conocimiento de las características y posibilidades del punto de venta, es todo un conjunto de acciones realizadas con una finalidad, el rendimiento del producto en la tienda. Según Mouton (2003), entre las razones para hacer Merchandising, se encuentran:

- Surtido: Es necesario adaptar el surtido en función de la zona de comercio, vocación y conocimiento de los mercados afectados.

- Implantación: Hay que implantar estanterías de venta en función del flujo de clientela y adaptar el mobiliario al surtido en la medida de lo posible.

- Presentación: Hay que hacer una presentación comercial de los productos al interior y saber establecer una secuencia lógica del consumidor de las familias y las subfamilias.

- Negociación: Hay que saber negociar con los proveedores, cuyos representantes suelen seguir unas etapas de venta, en la medida en que haya posibilidades de negociar con las tiendas. La negociación de compra es un acto importante y técnico.

- Potencial: Hay que conocer el potencial de venta del segmento determinado para una zona determinada.

- Rentabilidad: El espacio de venta representa una inversión, hay que rentabilizar cada metro cuadrado o lineal: es productividad aplicada a la distribución. Los análisis, regulares de rentabilidad, tienen que tener en cuenta todos los parámetros: Margen, estanterías, stocks, plazo de cobros y ventajas diferidas, a fin de efectuar cálculos reales y objetivos

- El Merchandising, opera ahora con la regla de los seis adecuados, que se refiere a los aspectos de gestión a tener en cuenta para realizar exitosamente sus acciones en el punto de venta: el producto adecuado, el tiempo adecuado, la cantidad adecuada, el precio adecuado y la forma de presentación adecuada.

El Merchandising visual, consiste en exhibir correctamente los productos determinando su lugar de ubicación en el lineal, con el fin de optimizar la circulación de la clientela dentro del establecimiento y para que, de esta forma, la compra resulte lo más cómoda y atractiva para 


\section{LA INCIDENCIA DEL MERCHANDISING EN EL CONTEXTO MUNDIAL}

el cliente y lo más rentable posible para el detallista. Su objetivo es dirigir el flujo de clientes hacia determinadas secciones o productos, provocar ventas por impulso, poner los productos al alcance del consumidor y diseñar el establecimiento de forma lógica. Asimismo, el Merchandising de gestión, consiste en rentabilizar el punto de venta determinando el tamaño óptimo del lineal, el desglose en las diversas familias, el número de referencias, marcas y caras expositoras del producto (ver tabla 1), (Bort, 2004, p. 28).

Tabla 1

Diagrama Merchandising visual

\begin{tabular}{ll}
\hline Disposición exterior & Escaparate \\
& Fachada \\
& Entrada \\
& Rótulo \\
& Orden interno \\
Trazado interior & Ubicación de secciones \\
& Visibilidad \\
Ambientación & Amplitud \\
& Color \\
Organización de mercancía & Elección de mobiliario \\
& Niveles de exposición \\
& Tipos de implementación \\
\hline
\end{tabular}

Fuente: Miguel A. Bort Muñoz, Merchandising.

El Merchandising de Gestión, según Bort (2004), en el estudio de mercado se debe recoger y analizar información de forma constante respecto al comportamiento de los consumidores, a la clientela habitual del establecimiento y a la competencia. Estos estudios, son necesarios para adecuar la oferta a las nuevas exigencias de los consumidores, modificar la oferta en función de las peticiones de los clientes, adaptar la política comercial del punto de venta al entorno en el que está ubicado el establecimiento y diferenciarse de los establecimientos que sean claros competidores. La gestión del espacio, consiste en colocar las secciones y los productos que integran cada una de estas de una forma adecuada para fomentar la venta obteniendo una mayor rentabilidad. En definitiva, se trata de optimizar el rendimiento del lineal y la gestión del espacio mediante la rotación, la rentabilidad y el beneficio efectuando comparaciones. La gestión del surtido a su vez, selecciona el surtido más adecuado al público objetivo estructurado en niveles tales como secciones, familias y subfamilias. La comunicación busca establecer el programa de comunicación de la empresa según su mercado. 
Tabla 2

Diagrama Merchandising de gestión

\begin{tabular}{ll}
\hline Estudios de mercado & $\begin{array}{l}\text { Consumidor } \\
\text { Clientes } \\
\text { Competencia }\end{array}$ \\
Gestión del surtido & Selección \\
Estructura \\
Gestión del espacio & $\begin{array}{l}\text { Optimización de línea } \\
\text { Gestión del espacio } \\
\text { Rotación }\end{array}$ \\
Comunicación & PVL \\
& Promociones \\
& Tipos de implementación \\
\hline
\end{tabular}

Fuente: Miguel A. Bort Muñoz, Merchandising.

El Merchandising en los establecimientos tradicionales colombianos, tiene un componente de gran peso y es el factor cultural, ya que el proceso de compra no se limita a la transacción comercial o la satisfacción de una necesidad; existe un factor de interacción social y cultural entre el vendedor y el cliente que le da un valor agregado a este tipo de compras. Palomares (2009), presenta una visión integral sobre los diferentes abordajes de la clasificación del Merchandising, según variables que la determinan, considerada como clasificación tradicional. El Merchandising de presentación, pretende que el lineal conduzca la atracción sobre los productos más rentables. Se utilizan medios como Publicidad en el Punto de venta (POP) y la ubicación de los productos. También, se intenta determinar la disposición interna de la tienda para optimizar la circulación de la clientela, así como en establecer el lugar de ubicación y el espacio asignado para los productos en el lineal de acuerdo a la selección, disposición y presentación del surtido. El Merchandising de seducción, se fundamenta en la tienda espectáculo, mobiliario específico, horarios, especialización, animación del punto de venta, productos vivos como los ecológicos, lights, entre otros. Busca promover la imagen del propio distribuidor, mediante un buen servicio y atención al cliente, cuida del aspecto del establecimiento e intenta lograr el mejor ambiente para influir en el ánimo de compra del consumidor. Por su parte, el Merchandising de gestión, implica realizar un análisis permanente de la oferta de la empresa detallista, con la idea de incrementar la rentabilidad y rotación de los productos en el punto de venta a través de la satisfacción de los consumidores. Se emplean instrumentos como estudios de mercado, Coeficiente de Ocupación del Suelo (COS), gestión de categorías, gestión de surtido, políticas de precio, posicionamiento y ubicación de los productos en el lineal. 


\section{LA INCIDENCIA DEL MERCHANDISING EN EL CONTEXTO MUNDIAL}

Hay que tener en cuenta quien es la persona encargada de realizar las acciones. El Merchandising del fabricante, se caracteriza por tener acciones en el punto de venta, por parte del fabricante de un producto orientadas, tanto al comprador, como al propio establecimiento o a su personal; para dar a conocer el producto, o para destacarlo de la competencia, con el objetivo de incrementar su rotación. El Merchandising del distribuidor, se caracteriza por desarrollar acciones en el establecimiento realizadas por el minorista, con el objeto de aumentar la venta de los productos, a fin de optimizar la rentabilidad de la superficie de venta. Asimismo, se debe analizar el tipo de cliente al que va dirigido. El Merchandising para el cliente-oportunista o también llamado Shopper, va dirigido a aquella persona que, para definir su intención de comprar, observa las alternativas que le confieren los diferentes establecimientos del mercado, tales como ubicación, comodidad, prestigio y demás factores que le brinden el beneficio que busca, el cual es necesario para impulsarle a consumir.

El Merchandising para el cliente-comprador, conocido como cliente Buyer se refiere a aquellos individuos que concretan la transacción de compra. Son también, aquellos que estando en el punto de venta adquieren los bienes o servicios dependiendo de los factores y beneficios que le impulsan a realizar la compra, tales como: precio, calidad, atención y surtido. También se desarrolla Merchandising de acuerdo con la vida del producto. Los productos, en relación al tiempo, atraviesan cuatro fases definidas por su ritmo de venta, que son: introducción, crecimiento, madurez y declive. Por esto, las acciones de Merchandising deben adecuarse a dichas etapas, dando la siguiente clasificación.

El Merchandising de nacimiento tiene que ver con los acuerdos de codificación o referenciación del producto, con su implantación en el lineal adecuado. En el punto de venta, se suelen realizar acciones como degustaciones, demostraciones, entrega de folletos, stands y ofertas de lanzamiento. El Merchandising de ataque, consiste en obtener un mejor emplazamiento en el lineal, como consecuencia de la mayor rotación y ventas del producto, con el objeto no sólo de lograr más clientes sino, también de fidelizar a los que ya se tienen. Cobran importancia las actividades de animación en el punto de venta y las campañas promocionales relacionadas con el producto. El Merchandising de mantenimiento, son las acciones que se llevan a cabo una vez que se ha obtenido el lineal adecuado al ritmo de venta y rotación del producto, con el fin de proteger ese emplazamiento, deben cultivarse las relaciones con los encargados de las tiendas y no descuidar las actividades de animación y promoción. Finalmente, el Merchandising de defensa, es la intervención que se realiza con el objeto de prolongar la fase de decadencia del producto, evitando la pérdida de posicionamiento en el lineal y procurando obtener la colaboración del distribuidor que, debido a la bajada en las ventas, pierde interés en la defensa del producto. 


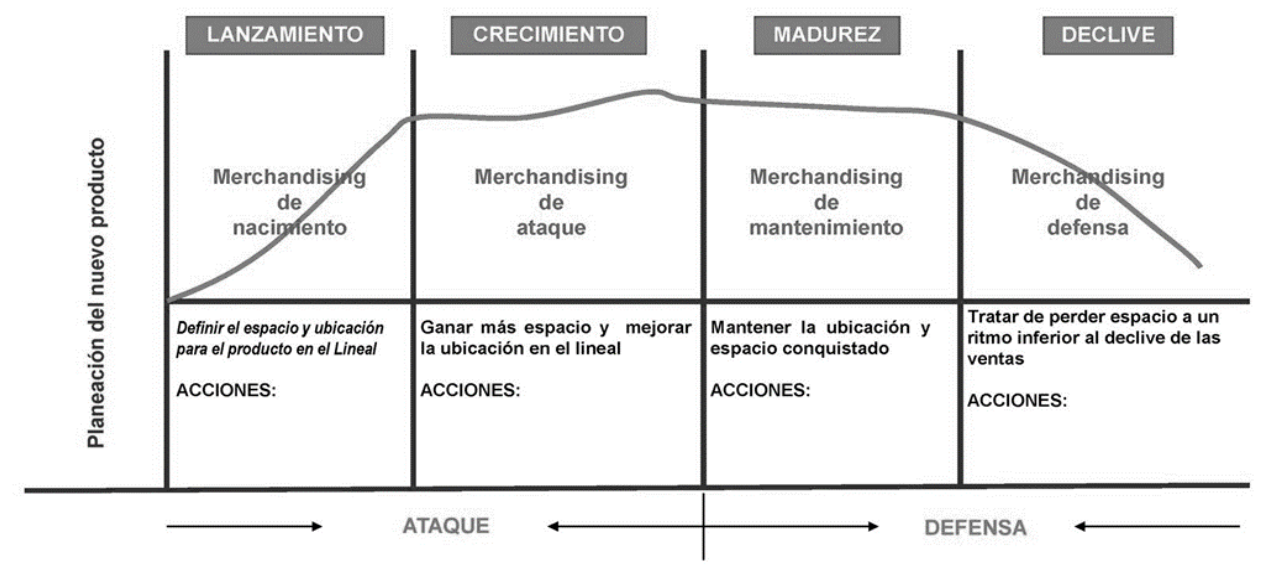

Figura 1. Acciones de Merchandising.

Fuente: Elaboración propia.

Para Palomares (2009), el Merchandising en función del tiempo hace referencia al Merchandising promocional constituido por el conjunto de acciones realizadas en un tiempo definido, para impulsar la compra, al Merchandising permanente que es la actividad continua ejecutada en el lineal, a lo largo de todo el año, el Merchandising estacional que se realiza en virtud de la relación de los productos con la época del año. Con lo anterior, se reviste la importancia del ambiente al momento de compra y según McGoldrick (1990), sostiene que el ambiente de venta es considerado como uno de los principales elementos de retail marketing mix y ello, desde varios puntos de vista: la relación efectiva de coste-espacio de la tienda, la consideración del espacio de venta como un todo para asegurar una experiencia de venta atractiva y, para conseguir una fuerte diferenciación de imagen. Así, el diseño de la tienda ha llegado a ser uno de los elementos más visibles del posicionamiento estratégico de la tienda.

Estudios basados en varios autores relacionados con la sicología ambiental, sugieren que la atmósfera de la tienda engendrada por un gran número de variables dentro de ella, es representada psicológicamente por los consumidores en términos de dos principales estados emocionales: placer y excitación. Estos dos estados emocionales son los importantes mediadores entre los estímulos dentro de la tienda y la conducta del comprador. Las respuestas de conducta están clasificadas en unas series de respuestas de acercamiento o evitación. (McGoldrick, 1990). Con base en lo anterior, se observa que las respuestas de consumo ante un producto vendrían determinadas no sólo por las interacciones que se dan entre el producto y el consumidor, sino también por la serie de interacciones que tienen lugar entre estos. A su vez y de acuerdo con Santesmases (2002), aspectos relacionados con el neuromarketing como la luz, el color, la temperatura, la música, los olores, etc. influyen en las percepciones de los compradores cuando visitan un establecimiento comercial y contribuyen a configurar la imagen del mismo. 


\section{LA INCIDENCIA DEL MERCHANDISING EN EL CONTEXTO MUNDIAL}

Un punto de venta es, sobre todo un escenario que permite poner en práctica los conocimientos de técnicas de marketing experiencial, de Merchandising, y de shopping (Underhill, 2000). Con este marco de referencia, se puede entender porque un punto de venta es más que su función comercial, es un punto de encuentro entre cultura y sujetos, como afirma Augé (1995). Esto se ve reforzado por Kotler (2006), quien indica que los consumidores en su proceso de decisión de compra, de productos o servicios, responden a más factores que los meramente tangibles. Así, los compradores responden al producto total que incluye sus servicios, garantías y entre todos ellos uno de los elementos principales es el lugar, más específicamente, las instalaciones y la atmósfera donde el producto es comprado o consumido, llegando en muchas ocasiones a ser esto el producto principal. Además, Kotler, (2006) estima que la estética espacial será usada dentro del marketing, como una parte más del marketing mix, de la misma forma a como se usa la variable precio, publicidad y relaciones públicas, sobre todo, esto será así en aquellos sectores en los que el número de establecimientos sean elevados, o en mercados en los que la diferencia de producto o precio sea muy pequeña. Por esto, se define el concepto atmósfera como el diseño del espacio creado conscientemente para generar determinados efectos en los compradores, más específicamente, es el esfuerzo para diseñar entornos de compra que produzcan efectos emocionales específicos en el comprador con el objetivo de potenciar su probabilidad de compra (Kotler, 2006).

En el pensamiento postmoderno, el espacio es un lugar de cambio que puede ser, además, un espacio de experiencia, de percepción o de imaginación por parte de una persona. Estas experiencias las ha clasificado, Schmitt (1999), en cinco tipos, también, llamados módulos experienciales estratégicos, que forman la base del sistema del marketing experiencial:

- Sensaciones: El marketing de sensaciones, apela a los sentidos, con el objeto de crear experiencias sensoriales a través de la vista, el oído, el tacto, el gusto y el olfato.

- Sentimientos: El marketing de sentimientos, apela a los sentimientos y a las emociones más internas de los clientes, con el objetivo de crear experiencias afectivas, que vayan desde estados de ánimo ligeramente positivos vinculados a una marca, hasta fuertes emociones de alegría y orgullo.

- Pensamientos: El marketing de pensamientos, apela al intelecto con objeto de crear experiencias cognitivas, que resuelvan problemas y que atraigan a los clientes creativamente.

- Actuaciones: El marketing de actuaciones, se propone afectar a experiencias corporales, estilos de vida e interacciones. El marketing de actuaciones, enriquece la 


\section{LA INCIDENCIA DEL MERCHANDISING EN EL CONTEXTO MUNDIAL}

vida de los clientes, ampliando sus experiencias físicas, mostrándoles formas alternativas de hacer cosas, y estilos de vida.

- Relaciones: El marketing de relaciones, contiene aspectos del marketing de sensaciones, sentimientos, pensamientos y actuaciones. Sin embargo, se extiende más allá, apelando al deseo de mejora del individuo, a la necesidad de ser percibidos de forma positiva por otras personas, estableciendo de este modo fuertes relaciones de marca.

El consumo es una experiencia, cada negocio un teatro. En la época de la economía de la experiencia, los propios consumidores se convierten en producto, por lo que ellos demandan experiencias que puedan transformar su conducta, su salud, incluso sus vidas. Cuando una persona compra una experiencia, compra un conjunto de actividades intangibles desarrolladas en su nombre, paga por gastar su tiempo en un divertido y memorable evento desarrollado en un escenario en el que está involucrado de forma personal. Todo tipo de empresas pueden hacer esto ya que las empresas deben reconocer que los productos y servicios ya no son suficientes, los consumidores quieren experiencias (Pine y Gilmore, 2001).

Con lo anterior, se indica que existen ocho pasos para destacar en el anaquel o la góndola de productos en exhibición, este plan de trabajo fue creado por el Food Marketing Institute de USA, en la década de los 80's con la finalidad de descubrir qué mejoras se podían hacer en las tiendas. En primera instancia se encuentra la definición de la categoría; es decir, qué es, qué o quiénes la conforman. Luego se define el rol de la categoría, en otras palabras, qué papel juega para el detallista. Seguidamente se evalúa la situación actual desde la perspectiva de cuánto vende hoy, con qué inventario se maneja, qué rentabilidad se tiene, cómo se está exhibiendo, cómo la compran los consumidores, cómo la ven. Se define el objetivo de la categoría en función de las ventas del próximo año y finalmente, se formulan las estrategias tomando en cuenta los objetivos y los planes para conseguirlos y las tácticas definidas en hechos por hacer para aplicar las estrategias y cumplir con los objetivos. Las tácticas pueden girar en torno al precio, analizando los precios de los productos de la categoría y la competencia, al surtido o portafolio eficiente de producto que se manejarán dentro de la categoría, a los productos o lanzamientos nuevos y el lugar en donde se va a colocar dentro de la tienda y finalmente el tipo de exhibición del anaquel en el planograma. Se deben acomodar los productos en el espacio que le corresponde, según las estrategias de la empresa; la idea es cuidar que los artículos que son importantes para la categoría tengan buena exhibición. 


\section{LA INCIDENCIA DEL MERCHANDISING EN EL CONTEXTO MUNDIAL}

En el contexto del Merchandising se denomina lineal a la superficie de stocks y de exposición de los productos en el lugar de venta. Las góndolas sobre las que se presentan los productos suelen tener una altura aproximada de 1,80 metros con cinco estanterías y un valor comercial muy desigual; las estanterías más altas y más bajas son las que tienen menor valor o las que menos ventas realizan. Las del medio, siendo las que mejor se ven y, sobre todo, las más accesibles a la mano del ama de casa son, con diferencia, la mejor situación de casi la totalidad de los productos. Se comprende fácilmente el interés de muchos fabricantes por situar sus productos en las mejores estanterías, intentando evitar que sea el distribuidor el que los sitúe según sus criterios de rentabilidad o, como hace algunas veces, al azar. Los productos se identifican perfectamente a través de la superficie del lineal, por lo que todas las empresas requieren conseguir mayores metros para la colocación de su producto. Se ubican en tres niveles: nivel de ojos, son los productos con mayor posibilidad de rotación; nivel manos, son los productos que por lo general son de consumo diario; y, nivel suelo, son los productos pesados y de uso regular.

La estantería más baja sirve, sobretodo, para productos de consumo diario, fácilmente identificables por el ama de casa (barriles de detergente, bolsas de patatas, entre otros). La disposición de los productos en las góndolas, deben cumplir el principio fundamental del Merchandising, que dice lo siguiente: "Todo lo que se ve, se vende; todo lo que se coge, se compra" (Ricoveri, 2003).

\section{CONSIDERACIONES E IMPLICACIONES}

Este artículo procura dar una visión integral del desarrollo del Merchandising como técnica de exhibición comercial, desde una perspectiva teórica. Con esta revisión, se aprecia la importancia de consolidar los elementos que desde la teoría pueden tener relevancia práctica y ser desarrolladas por las empresas en los diversos entornos donde esta opere, permitiendo tener un impacto positivo. Por tal razón, toda propuesta de Merchandising debe incluir como mínimo objetivos de ubicación, espacio y rentabilidad; argumentos de ventas extraídos de la publicidad; conocimiento del producto en cuanto a su marca, posicionamiento, composición, sabores, colores, empaques y tamaños; tipos de herramientas como exhibiciones adicionales, material P.O.P.; zonas geográficas y duración de los eventos si han de ser permanentes, de temporada o promocionales. Igualmente, la identificación del tipo de negocio, si es grande superficie, autoservicio, tienda tradicional; localización de espacios, instrumentos de comunicación y promoción; tipos de exhibición, de evaluación y de seguimiento. Cada organización debe conocer las tendencias y los hechos que han llevado las ventas al por menor que se han ido desarrollando en especial en países de América Latina, tales como: la 


\section{LA INCIDENCIA DEL MERCHANDISING EN EL CONTEXTO MUNDIAL}

crisis acelerada de la economía mundial, la competencia fuerte por el mercado de cadenas y distribuidores, los grandes esfuerzos por la fidelización del consumidor, el fracaso del desarrollo de algunos puntos de venta, la desaparición de los formatos tradicionales, el auge de nuevos formatos importados de países desarrollados, la creciente sinergia entre fabricantes y distribuidores y el incremento de las expectativas del consumidor.

Hay que tener en cuenta que hoy el Merchandising aplicado de manera adecuada es efectivo y rápido. Se necesita de innovación en la promoción y en la publicidad, dejando de lado los medios tradicionales como televisión, radio o prensa. Los clientes cada vez están más informados, surgen temas relevantes y paralelos como el consumo responsable y con él la responsabilidad social empresarial sumada a la innovación en la tecnología. Se deben incorporar al Merchandising los aspectos creativos de la promoción y la publicidad de la empresa, investigar en el campo qué herramientas de Merchandising se requieren para mejorar le afectividad en las ventas; así como sacar mayor provecho de los materiales de Merchandising en el último momento.

\section{CONCLUSIONES}

En muchos aspectos, la importancia del Merchandising radica en su capacidad de manipular psicológicamente al cliente. En última instancia, más allá de la comida, agua y ropa, ningún producto que se encuentre en un establecimiento comercial constituye una necesidad para el cliente. Cuando se obtiene el éxito, el impacto psicológico de las unidades de Merchandising lleva a las ventas, genera conocimiento del producto, crea un entorno visual confortable para el cliente y bombea dinero en el punto de venta. También puede afectar a la forma de ver ciertos productos, influyendo así en las futuras decisiones de compra. Finalmente, se invita a hacer una revisión y análisis del término Merchandising, para que, en futuras investigaciones, se pueda determinar la puesta en práctica de esta técnica de la manera más adecuada.

\section{REFERENCIAS BIBLIOGRÁFICAS}

Augé, M. (1995): Los “no lugares”. Espacios del anonimato. Barcelona: Gedisa.

Bort, M. (2004) Merchandising: Cómo mejorar la imagen de un establecimiento comercial. Madrid: ESIC Editorial.

Fresco, J. (1999). Marketing desde el punto de venta: Merchandising: casos prácticos. Argentina: Macchi.

Kepner, C. (1992): Merchandising avos marques. Presses du Management, Paris: Noisiel. 
LA INCIDENCIA DEL MERCHANDISING EN EL CONTEXTO MUNDIAL

Kerlinger. F. (2002). Investigación del Comportamiento. México: McGraw-Hill.

Kotler, P. (1973). El marketing según Kotler. Barcelona: Paidós.

Kotler, P. (1993). Dirección de Mercadotecnia (7a ed.). México. D.F.: Prentice-Hall.

Kotler, P. (2006). Dirección de Mercadotecnia (12ª ed.). México, D.F.: Prentice-Hall.

McGoldrick, P. (1990). Retail Marketing. London: McGraw-Hill.

Mouton, D. (2003). Merchandising estratégico. Barcelona: Ediciones Gestión 2.000.

Palomares, R. (2001). Merchandising. Cómo vender más en establecimientos comerciales, Barcelona: Ediciones Gestión 2000.

Palomares, R. (2009). Merchandising. Teoría, práctica y estrategia, Madrid: ESIC Editorial.

Palomares, R. (2013). Marketing en el punto de venta, Madrid, España: ESIC Editorial.

Prieto, J. (2010). Merchandising, la seducción en el punto de venta (2a ed.). México: Ecoe Ediciones.

Pine II, B. y Gilmore, J. (1999). The Experience Economy, Estados Unidos: Harvard Business Review Press

Sánchez, J. (1997). Marketing financiero. Principios y Estrategias. Madrid: Civitas.

Santesmases, M. (2002). Usted compra, yo vendo: ¿Qué tenemos en común? Madrid: Pirámide.

Schmitt, B. (1999): Experiential Marketing. Barcelona: Deusto S. A. Ediciones.

Teruel, M. (1995). Marketing financiero y de servicios de la oficina bancaria. Madrid: Universidad Pontificia Comillas.

Underhill, P. (2000). Porque compramos, La ciencia del shopping. Barcelona: Ediciones Gestión 2000

Wellhof, A. y Masson, J. (1999). El Merchandising. Barcelona: Deusto S. A. Ediciones 